\title{
Sycosiform tinea barbae caused by trichophyton rubrum and its association with autoinoculation ${ }^{*}$
}

\author{
Karina Colossi Furlan ${ }^{1}$ \\ Juliana Cabral Nunes Chartuni ${ }^{2}$
}

DOI: http:/ / dx.doi.org/10.1590/abd1806-4841.20174802

\author{
Priscila Kakizaki ${ }^{1}$ \\ Neusa Yuriko Sakai Valente ${ }^{1,3}$
}

\begin{abstract}
(
Dear Editor,

Tinea barbae is a dermatophyte infection that affects primarily the bearded areas of the face.1 Sycosis is one type of clinical manifestation associated with deep folliculitis. The infection is usually caused by zoophilic fungi acquired after contact with animals. 2 The presence of anthropophilic fungi, such as Trichophyton rubrum (T. rubrum), has recently been reported, and they often colonize nails.3,4 Since its first report by Nenoff et al.4 in 2008, the number of cases has grown, especially those involving nails, feet, or hands.5 Brazil reported one case of tinea barbae caused by T. rubrum in $2008^{1}$, but without autoinoculation. This case report highlights such an association and recommends attention for this diagnosis. A 68-year-old male patient showed suppurative nodular lesions for five months in his beard and on the right side of his neck

(Figure 1). He denied any previous treatments. Onycholysis and hyperkeratosis affected all of the nail plate on his first right toe, but his fingernails apparently remained intact. Exams from culture and direct examination of his beard hair and the suppurative material from the lesion identified T. rubrum fungus (Figure 2). Incisional biopsy of a suppurative lesion showed acute suppurative folliculitis; slide analysis in immersion showed hyphae within the hair (endotrix) and conidium in the perifollicular zone (ectotrix) (Figure 3). Analysis of nail scrapes through direct examination by microscope with potassium hydroxide showed dermatophytes; culture testing showed T. rubrum growth. The treatment chosen was oral itraconazole $200 \mathrm{mg}$ / day for 30 days, which was effective for the lesions on the face and for residual hyperpigmentation.
\end{abstract}

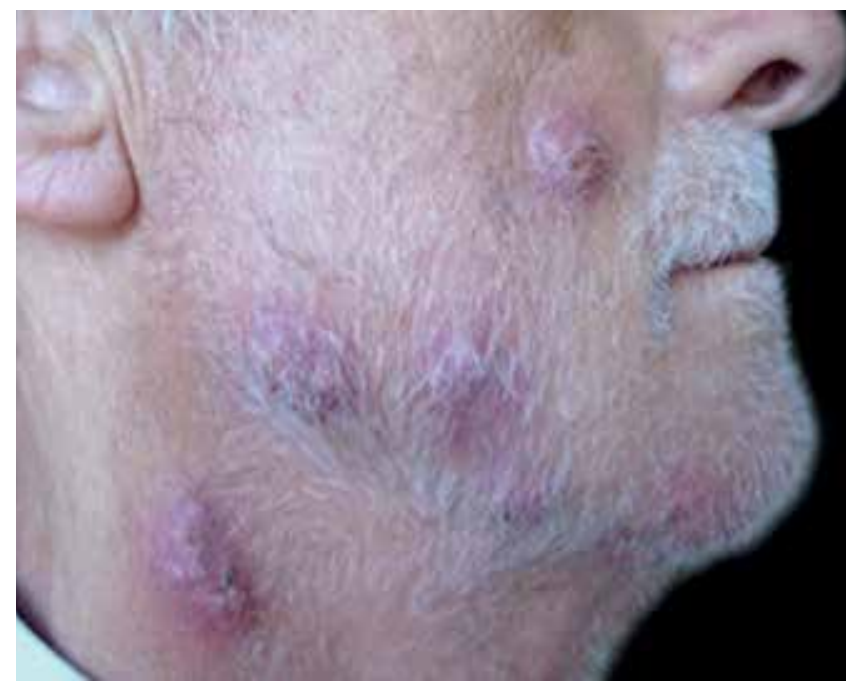

Figure 1: Sycosiform lesions on the beard (right side)

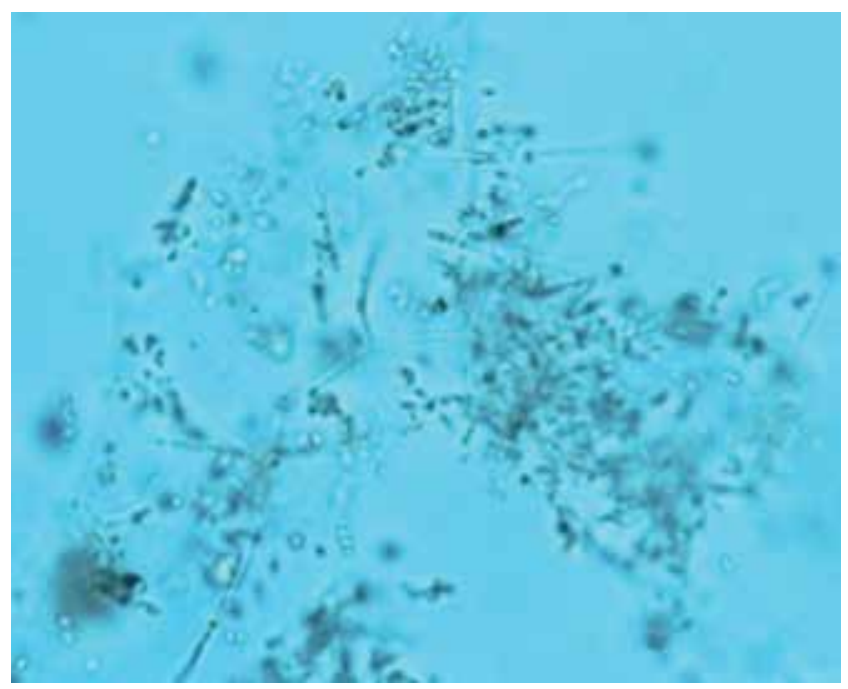

FIGURE 2: Micromorphological aspect of T. rubrum colony

\section{Received on 04.06.2015}

Approved by the Advisory Board and accepted for publication on 08.08.2015

* Work performed at Hospital do Servidor Público Estadual de São Paulo - São Paulo (SP), Brazil.

Financial Support: None.

Conflict of Interest: None.

Hospital do Servidor Público Estadual de São Paulo - São Paulo (SP), Brazil.

Hospital Santa Maria - Brasília (DF), Brazil.

Faculdade de Medicina da Universidade de São Paulo (USP) - São Paulo (SP), Brazil.

C2017 by Anais Brasileiros de Dermatologia 


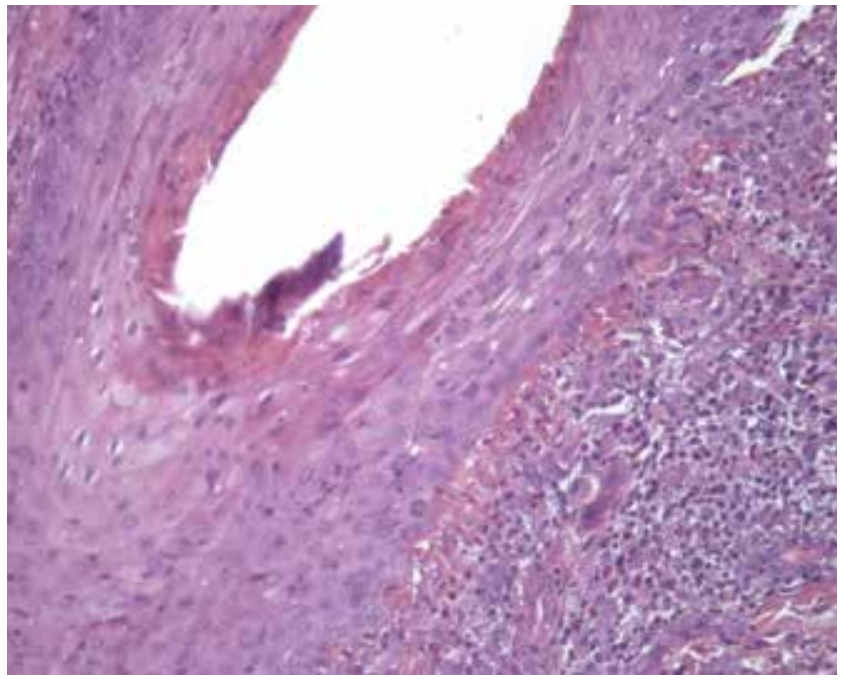

Figure 3: Histopathology showing inflammattory suppurative perifollicular (hematoxylin and eosin)

Tinea barbae, caused by Trichophyton verrucosum and Trichophyton mentagrophytes, usually affects farmers and farmworkers. The anthropophilic species Trichophytum violaceum, Trichophytum schoenleinii, Trichophytum megninii, and T. rubrum are occasional agents and affect the hair shaft.4 Lesions are probably triggered by autoinoculation, which is usually observed after local trauma caused by scratch or, more commonly, by shaving razors. Clinically, tinea barbae may be inflammatory or non-inflammatory, depending on the fungus and the patient's immune response. Deep folliculitis is inflammatory; its clinical lesions are follicular pustules and fistula on the skin surface. 3 The non-inflammatory subtype is superficial and similar to tinea corporis or superficial folliculitis. The diagnosis is made by direct examination of skin samples, followed by culture and microculture testing. The fungus has been found ectotrix and endotrix, confirming penetration into the hair shaft. Tinea barbae should be treated with oral antifungal medications, similarly to scalp ringworm, as the inner hair shaft is also affected. In this case, Griseofulvin is the only drug approved by the Food and Drug Administration (FDA). The dose prescribed is $500 \mathrm{mg}$ to $1000 \mathrm{mg} /$ day for 6 to 12 weeks. Terbinafina $250 \mathrm{mg} /$ day for 4 weeks is the treatment of choice for many authors.3 Oral itraconazole $200 \mathrm{mg} /$ day for 4 to 6 weeks may also be prescribed. T. rubrum is an anthropophilic fungus that usually causes tinea pedis and onychomycosis. The literature has reported tinea barbae caused by T. rubrum associated with onychomycosis, or tinea pedis by the same fungus. The case reported here showed this association with a unique clinical manifestation. It is still unclear whether such an association is rare; however, as the tinea barbae is an unusual ringworm caused primarily by zoophilic fungi, we emphasize the rarity of the lesion and its possible association with autoinoculation.

\section{REFERENCES}

1. Xavier MH, Torturella DM, Rehfeldt FV, Alvariño CR, Gaspar NN, Rochael MC, et al. Sycosiform tinea barbae caused by Trichophyton rubrum. Dermatol Online J. 2008;14:10.

2. Sabota J, Brodell R, Rutecki GW, Hoppes WL. Severe tinea barbae due to Trichophyton verrucosum infection in dairy farmers. Clin Infect Dis. 1996;23:1308-10.

3. Szepietowski JC, Matusiak L. Trichophyton rubrum autoinoculation from infected nails is not such a rare phenomenon. Mycoses. 2008;51:345-6.

4. Kawada A, Aragane Y, Maeda A, Yudate T, Tezuka T, Hiruma M. Tinea barbae due to Trichophyton rubrum with possible involvement of autoinoculation. Br J Dermatol. 2000;142:1064-5.

5. Nenoff P1, Mügge C, Herrmann J, Keller U. Tinea faciei incognito due to Trichophyton rubrum as a result of autoinoculation from onychomycosis. Mycoses. 2007;50:20-5.

\author{
MAILING ADDRESS: \\ Karina Colossi Furlan \\ Av. Ibirapuera, 981 \\ Indianópolis \\ 04028-000 São Paulo, SP - Brazil \\ E-mail:kcolossi@gmail.com \\ ju_cabral_18@hotmail.com \\ neusasvalente@gmail.com
}

How to cite this article: Furlan KC, Kakizaki P, Chartuni JCN, Valente NYS. Sycosiform tinea barbae caused by Trichophyton rubrum and its association with autoinoculation An Bras Dermatol. 2017;92(1):160-1. 